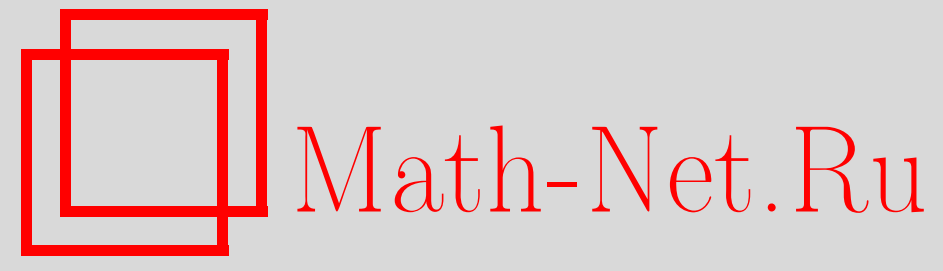

E. A. Feinberg, P. O. Kasyanov, N. V. Zadoianchuk, Fatou's lemma for weakly converging probabilities, Теория вероятн. и ее примен., 2013, том 58, выпуск 4, 812-818

DOI: https://doi.org/10.4213/tvp4544

Использование Общероссийского математического портала Math-Net.Ru подразумевает, что вы прочитали и согласны с пользовательским соглашением http://www . mathnet.ru/rus/agreement

Параметры загрузки:

IP : 54.84 .234 .179

26 апреля 2023 г., 15:05:07

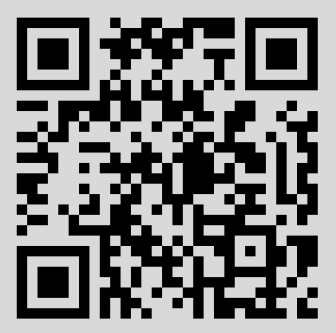


12. Louhichi $S$. Weak convergence for empirical processes of associated sequences. - Ann. Inst. Henri Poincare, Probabilites et Statistiques, 2000, v. 36, №, p. 547-567.

Поступила в редакцию 24.X.2012

(C) 2013 г.

FEINBERG E.A.* KASYANOV P.O.**, ZADOIANCHUK N. V.**

\title{
FATOU'S LEMMA FOR WEAKLY CONVERGING PROBABILITIES ${ }^{1)}$
}

\begin{abstract}
Лемма Фату утверждает при определенных условиях, что интеграл нижнего предела последовательности функций не превышает нижнего предела соответствующих интегралов. Данная заметка описывает подобные неравенства, когда, в отличие от случая одной меры, функции интегрируются относительно разных мер, которые образуют слабо сходящуюся последовательность.
\end{abstract}

Ключевые слова и фразы: лемма Фату, вероятность, мера, слабая сходимость.

1. The inequality for nonnegative functions. Consider a measurable space $(S, \mathscr{B})$, where $S$ is a metric space and $\mathscr{B}$ is its Borel $\sigma$-field. Let $\mathbf{P}(S)$ be the set of probability measures on $(S, \mathscr{B}(S))$. According to Fatou's lemma, [8], for any $\mu \in \mathbf{P}(S)$ and for any sequence of nonnegative measurable functions $f_{1}, f_{2}, \ldots$

$$
\int_{S} \liminf _{n \rightarrow+\infty} f_{n}(s) \mu(d s) \leqslant \liminf _{n \rightarrow+\infty} \int_{S} f_{n}(s) \mu(d s) .
$$

A sequence of probability measures $\left\{\mu_{n}\right\}_{n \geqslant 1}$ from $\mathbf{P}(S)$ converges weakly to $\mu \in \mathbf{P}(S)$ if for any bounded continuous function $f$ on $S$

$$
\int_{S} f(s) \mu_{n}(d s) \rightarrow \int_{S} f(s) \mu(d s) \quad \text { as } n \rightarrow+\infty .
$$

A sequence of probability measures $\left\{\mu_{n}\right\}$ from $\mathbf{P}(S)$ converges setwise to $\mu \in \mathbf{P}(S)$ if (1.2) holds for any bounded measurable function $f$. If $\left\{\mu_{n}\right\}_{n \geqslant 1} \subset \mathbf{P}(S)$ converges setwise to $\mu \in \mathbf{P}(S)$, according to Royden [5, p. 231], for any sequence of nonnegative measurable function $f_{1}, f_{2}, \ldots$

$$
\int_{S} \liminf _{n \rightarrow+\infty} f_{n}(s) \mu(d s) \leqslant \liminf _{n \rightarrow+\infty} \int_{S} f_{n}(s) \mu_{n}(d s) .
$$

However, this is not true, if $\mu_{1}, \mu_{2}, \ldots$ converge weakly to $\mu$.

Indeed, let $S=[0,1], \mu_{n}(A)=\mathbf{I}\{1 / n \in A\}, \mu(A)=\mathbf{I}\{0 \in A\}$ for $A \in \mathscr{B}([0,1])$, and $f(s)=f_{n}(s)=\mathbf{I}\{s=0\}$ for $n=1,2, \ldots$ and $s \in[0,1]$. Then $\int_{S} f(s) \mu(d s)=1$, $\int_{S} f(s) \mu_{n}(d s)=0$, and (1.3) does not hold.

Theorem 1.1 presents Fautou's lemma for weakly converging measures $\mu_{n}$ and nonnegative functions $f_{n}$. This fact is useful for the analysis of Markov decision processes

* Department of Applied Mathematics and Statistics, Stony Brook University, Stony Brook, NY 11794-3600, USA; e-mail: eugene.feinberg@sunysb.edu

** Institute for Applied System Analysis, National Technical University of Ukraine «Kyiv Polytechnic Institute», Peremogy ave., 37, Build. 35, 03056, Kyiv, Ukraine; e-mail: kasyanov@i.ua; ninellll@i.ua

1) This research was partially supported by NSF grants CMMI-0928490 and CMMI1335296, by the Ukrainian State Fund for Fundamental Research under grant GP/F49/070, and by grant 2273/13 from the National Academy of Sciences of Ukraine. 
and stochastic games. Serfozo [7, Lemma 3.2] establishes inequality (1.4) for a vaguelly convergent sequence of measures on a locally compact metric space $S$ and for nonnegative functions $f_{n}$. In its current form, Theorem 1.1 is formulated in [6, Lemma 2.3(ii)] without proof, in [4, Lemma 3.2] with short explanations on how the proof from [7, Lemma 3.2] can be adapted to weak convergence on metric spaces, and in [3, Lemma 4] with a proof. To make this note logically complete, we provide the proof of Theorem 1.1 in Section 3. The provided proof is shorter and simpler than the proof in [3]. Theorem 4.2 below extends Theorem 1.1 to functions $f_{n}$ that can be unbounded from below. Lemma 3.3 in [4] is a particular version of such a result developed for particular applications in that paper. Let $\overline{\mathbf{R}}=[-\infty,+\infty]$.

Theorem 1.1. Let $S$ be an arbitrary metric space, $\left\{\mu_{n}\right\}_{n \geqslant 1} \subset \mathbf{P}(S)$ converge weakly to $\mu \in \mathbf{P}(S)$, and $\left\{f_{n}\right\}_{n \geqslant 1}$ be a sequence of measurable nonnegative $\overline{\mathbf{R}}$-valued functions on $S$. Then

$$
\int_{S} \liminf _{n \rightarrow+\infty, s^{\prime} \rightarrow s} f_{n}\left(s^{\prime}\right) \mu(d s) \leqslant \liminf _{n \rightarrow+\infty} \int_{S} f_{n}(s) \mu_{n}(d s) .
$$

We remark that, if $f_{n}(s)=f(s), n=1,2, \ldots$, and the function $f$ is nonnegative and lower semicontinuous, then $\liminf _{n \rightarrow+\infty, s^{\prime} \rightarrow s} f_{n}\left(s^{\prime}\right)=f(s)$ and Theorem 1.1 implies that

$$
\int_{S} f(s) \mu(d s) \leqslant \liminf _{n \rightarrow+\infty} \int_{S} f(s) \mu_{n}(d s)
$$

if $\mu_{n}$ converges weakly to $\mu$; see [1, Problem 7, Chap. 1, §2], where this fact is stated for a bounded lower semicontinuous $f$.

Further, for any $\overline{\mathbf{R}}$-valued function $u$ on $S$ we denote

$$
\underline{u}(s)=\liminf _{s^{\prime} \rightarrow s} u\left(s^{\prime}\right), \quad \bar{u}(s)=\limsup _{s^{\prime} \rightarrow s} u\left(s^{\prime}\right), \quad s \in S .
$$

Theorem 4.2 provides the extended version of Theorem 1.1 for unbounded from below functions.

\section{Proof of Theorem 1.1.}

$\mathrm{P}$ r o o f. First, we prove the theorem for uniformly bounded from above functions $f_{n}$. Let $f_{n}(s) \leqslant K<+\infty$ for all $n=1,2, \ldots$ and all $s \in S$. For $n=1,2, \ldots$ and $s \in S$, define $F_{n}(s)=\inf _{m \geqslant n} f_{m}(s)$. The functions $\underline{F}_{n}: S \rightarrow[0,+\infty], n=1,2, \ldots$, are lower semi-continuous; see [2, Lemma 5.13.4]. In addition, for $s \in S$

$$
\underline{F}_{n}(s) \uparrow \liminf _{n \rightarrow+\infty, s^{\prime} \rightarrow s} f_{n}\left(s^{\prime}\right) \quad \text { as } n \rightarrow+\infty .
$$

By the monotone convergence theorem,

$$
\int_{S} \liminf _{n \rightarrow+\infty, s^{\prime} \rightarrow s} f_{n}\left(s^{\prime}\right) \mu(d s)=\lim _{n \rightarrow+\infty} \int_{S} \underline{F}_{n}(s) \mu(d s) .
$$

Since the function $\underline{F}_{n}, n=1,2, \ldots$, is lower semi-continuous on $S$ and bounded from below and $\mu_{m}$ converges weakly to $\mu$ as $m \rightarrow+\infty$, then formula (1.5) provides

$$
\int_{S} \underline{F}_{n}(s) \mu(d s) \leqslant \liminf _{m \rightarrow+\infty} \int_{S} \underline{F}_{n}(s) \mu_{m}(d s), \quad n=1,2, \ldots
$$

Since $\underline{F}_{n}$ is monotonically nondecreasing in $n=1,2, \ldots$, it follows that

$$
\liminf _{m \rightarrow+\infty} \int_{S} \underline{F}_{n}(s) \mu_{m}(d s) \leqslant \liminf _{m \rightarrow+\infty} \int_{S} \underline{F}_{m}(s) \mu_{m}(d s), \quad n=1,2, \ldots
$$

Formulas (2.2)-(2.4) provide necessary inequality (1.4).

Thus Theorem 1.1 is proved for uniformly bounded functions $f_{n}$. Consider a sequence $\left\{f_{n}\right\}_{n \geqslant 1}$ of measurable nonnegative $\overline{\mathbf{R}}$-valued functions on $S$. For $\lambda>0$ set $f_{n}^{\lambda}(s):=$ $\min \left\{f_{n}(s), \lambda\right\}, s \in S, n=1,2, \ldots$. Since the functions $f_{n}^{\lambda}$ are uniformly bounded from above,

$$
\int_{S} \liminf _{n \rightarrow+\infty, s^{\prime} \rightarrow s} f_{n}^{\lambda}\left(s^{\prime}\right) \mu(d s) \leqslant \liminf _{n \rightarrow+\infty} \int_{S} f_{n}^{\lambda}(s) \mu_{n}(d s) \leqslant \liminf _{n \rightarrow+\infty} \int_{S} f_{n}(s) \mu_{n}(d s) .
$$


Then, using Fatou's lemma,

$$
\int_{S} \liminf _{n \rightarrow+\infty, s^{\prime} \rightarrow s} f_{n}\left(s^{\prime}\right) \mu(d s) \leqslant \liminf _{\lambda \rightarrow+\infty} \int_{S} \liminf _{n \rightarrow+\infty, s^{\prime} \rightarrow s} f_{n}^{\lambda}\left(s^{\prime}\right) \mu(d s) .
$$

Theorem 1.1 is proved.

3. A counterexample for functions unbounded from below. A suitable assumption concerning the negative parts of the sequence $f_{1}, f_{2}, \ldots$ of functions is necessary for Fatou's lemma for weakly converging probabilities as well as for setwise converging probabilities, as the following example shows.

$\mathrm{E} \times \mathrm{a} \mathrm{m} \mathrm{p} \mathrm{le} \mathrm{3.1.} \mathrm{The} \mathrm{sequence} \mathrm{of} \mathrm{probability} \mathrm{measures}\left\{\mu_{n}\right\}_{n \geqslant 1}$ converges setwise (and therefore converges weakly) to a probability measure $\mu$ from $\mathbf{P}(S)$, a real function $f: S \rightarrow \mathbf{R}$ is continuous,

$$
\int|f(s)| \mu(d s), \quad \int|f(s)| \mu_{n}(d s)<+\infty, \quad n \geqslant 1,
$$

and

$$
\int f(s) \mu(d s)>\lim _{n \rightarrow+\infty} \int f(s) \mu_{n}(d s) .
$$

Let $S$ denote the semiinterval $(0,1]$ with the Borel $\sigma$-field $\mathscr{B}(S)$. For every natural number $n$ define the probability measure

$$
\mu_{n}(A)=\sqrt{n} \lambda\left(A \cap\left[\frac{1}{2 n}, \frac{1}{n}\right]\right)+\left(2-\frac{1}{\sqrt{n}}\right) \lambda\left(A \cap\left[\frac{1}{2}, 1\right]\right), \quad A \in \mathscr{B}(S),
$$

where $\lambda$ is the Lebesgue measure on $(0,1]$. Define also continuous on $S$ real function $f(s)=$ $-s^{-1}$. The sequence of probability measures $\left\{\mu_{n}\right\}_{n} \geqslant 1$ converges setwise (and therefore converges weakly) to the probability measure $\mu$ from $\mathbf{P}(S)$, where $\mu(A)=2 \lambda(A \cap[1 / 2,1])$, $A \in \mathscr{B}(S)$, and

$$
\int f(s) \mu(d s)=-2 \ln (2), \quad \int f(s) \mu_{n}(d s)=-\ln (2)\left(\sqrt{n}+2-\frac{1}{\sqrt{n}}\right), \quad n \geqslant 1 .
$$

Thus

$$
\int f(s) \mu(d s)>\lim _{n \rightarrow+\infty} \int f(s) \mu_{n}(d s)=-\infty .
$$

R e m a r $\mathrm{k} 3.1$. If we set $f(s)=s^{-1}$ for $s \in(0,1], n \geqslant 1$, in example 3.1, then inequalities (1.3) and (1.4) are strict.

4. Extensions and variations. In the rest of this paper, we deal with integrals of functions that can take negative values. An integral $\int_{S} f(s) \mu(d s)$ of a measurable $\overline{\mathbf{R}}$-valued function $f$ on $S$ with respect to a probability measure $\mu \in \mathbf{P}(S)$ is defined if

$$
\min \left\{\int_{S} f^{+}(s) \mu(d s), \int_{S} f^{-}(s) \mu(d s)\right\}<+\infty,
$$

where $f^{+}(s)=\max \{f(s), 0\}, f^{-}(s)=-\min \{f(s), 0\}, s \in S$. If (4.1) holds, then the integral is defined as

$$
\int_{S} f(s) \mu(d s)=\int_{S} f^{+}(s) \mu(d s)-\int_{S} f^{-}(s) \mu(d s) .
$$

All the integrals in the assumptions of the following theorems and corollary are assumed to be defined. For example, by writing (4.2) in Theorem 4.1, we assume that the integrals are defined for the functions $g_{n}(s), n \geqslant 1$, and $\lim \sup _{n \rightarrow+\infty} g_{n}(s)$. values.

The following statement is a generalization of (1.3) to functions that can take negative 
Theorem 4.1. Let $\left\{\mu_{n}\right\}_{n \geqslant 1} \subset \mathbf{P}(S)$ converge setwise to $\mu \in \mathbf{P}(S)$ and let $\left\{f_{n}\right\}_{n} \geqslant 1$ be a sequence of measurable $\overline{\mathbf{R}}$-valued functions defined on $(S, \mathscr{B}(S))$. Then inequality (1.3) holds, if all the integrals in (1.3) are defined and there exists a sequence of measurable $\mathbf{R}$-valued functions $\left\{g_{n}\right\}_{n \geqslant 1}$ on $S$ such that $f_{n}(s) \geqslant g_{n}(s)$, for all $n \geqslant 1$ and for all $s \in S$, and

$$
-\infty<\int_{S} \limsup _{n \rightarrow+\infty} g_{n}(s) \mu(d s) \leqslant \liminf _{n \rightarrow+\infty} \int_{S} g_{n}(s) \mu_{n}(d s) .
$$

$\mathrm{P} r$ o o f. If at least one of the inequalities

$$
\liminf _{n \rightarrow+\infty} \int_{S} f_{n}(s) \mu_{n}(d s)<+\infty, \quad-\infty<\int_{S} \liminf _{n \rightarrow+\infty} f_{n}(s) \mu(d s)
$$

is violated, then inequality (1.3) holds. So, we assume (4.3). The left inequality in (4.3) implies

$$
\liminf _{n \rightarrow+\infty} \int_{S} g_{n}(s) \mu_{n}(d s)<+\infty .
$$

Let us apply Fatou's lemma for setwise converging probabilities (see (1.3)) to the sequence $\left\{f_{n}-g_{n}\right\}_{n} \geqslant 1$ of nonnegative $\overline{\mathbf{R}}$-valued measurable functions on $S$. Then

$$
\int_{S} \liminf _{n \rightarrow+\infty}\left(f_{n}(s)-g_{n}(s)\right) \mu(d s) \leqslant \liminf _{n \rightarrow+\infty} \int_{S}\left(f_{n}(s)-g_{n}(s)\right) \mu_{n}(d s) .
$$

Inequalities (4.2) and (4.4) imply

$$
-\infty<\int_{S} \limsup _{n \rightarrow+\infty} g_{n}(s) \mu(d s)<+\infty .
$$

In view of (4.6) and the right inequality in (4.3),

$$
\liminf _{n \rightarrow+\infty} f_{n}(s)-\limsup _{n \rightarrow+\infty} g_{n}(s) \leqslant \liminf _{n \rightarrow+\infty}\left(f_{n}(s)-g_{n}(s)\right) \quad \mu(d s) \text {-a.s. },
$$

and

$$
\int_{S} \liminf _{n \rightarrow+\infty} f_{n}(s) \mu(d s)-\int_{S} \limsup _{n \rightarrow+\infty} g_{n}(s) \mu(d s) \leqslant \int_{S} \liminf _{n \rightarrow+\infty}\left(f_{n}(s)-g_{n}(s)\right) \mu(d s) .
$$

The following inequalities and (4.6) imply (1.3) since

$$
\begin{aligned}
\int_{S} \liminf _{n \rightarrow+\infty} & f_{n}(s) \mu(d s)-\int_{S} \limsup _{n \rightarrow+\infty} g_{n}(s) \mu(d s) \\
\leqslant & \liminf _{n \rightarrow+\infty} \int_{S}\left(f_{n}(s)-g_{n}(s)\right) \mu_{n}(d s) \\
\leqslant & \liminf _{n \rightarrow+\infty} \int_{S} f_{n}(s) \mu_{n}(d s)-\liminf _{n \rightarrow+\infty} \int_{S} g_{n}(s) \mu_{n}(d s) \\
\leqslant & \liminf _{n \rightarrow+\infty} \int_{S} f_{n}(s) \mu_{n}(d s)-\int_{S} \limsup _{n \rightarrow+\infty} g_{n}(s) \mu(d s),
\end{aligned}
$$

where the first inequality follows from (4.8) and (4.5), the second one holds since $-\infty<$ $\liminf _{n \rightarrow+\infty} \int_{S} g_{n}(s) \mu_{n}(d s)<+\infty$ in view of (4.2), (4.3), and $g_{n} \leqslant f_{n}$, and the last inequality holds because of (4.2) and (4.6). Theorem 4.1 is proved.

$\mathrm{R}$ e $\mathrm{m}$ a $\mathrm{rk}$ 4.1. The second inequality in (4.2) coincides with (1.3), when $f_{n}=$ $g_{n}=g, n=1,2, \ldots$.

The following theorem extends Theorem 1.1 to functions that can take negative values.

Theorem 4.2. Let $S$ be an arbitrary metric space, $\left\{\mu_{n}\right\}_{n \geqslant 1} \subset \mathbf{P}(S)$ converge weakly to $\mu \in \mathbf{P}(S)$, and $\left\{f_{n}\right\}_{n \geqslant 1}$ be a sequence of measurable $\overline{\mathbf{R}}$-valued functions on $S$. Then inequality (1.4) holds, if all the integrals in (1.4) are defined and there exists a sequence of measurable $\mathbf{R}$-valued functions $\left\{g_{n}\right\}_{n} \geqslant 1$ on $S$ such that $f_{n}(s) \geqslant g_{n}(s)$, for all $n \geqslant 1$ and for all $s \in S$, and

$$
-\infty<\int_{S} \limsup _{n \rightarrow+\infty, s^{\prime} \rightarrow s} g_{n}\left(s^{\prime}\right) \mu(d s) \leqslant \liminf _{n \rightarrow+\infty} \int_{S} g_{n}(s) \mu_{n}(d s) .
$$


$\mathrm{P} r$ o o f. If at least one of the inequalities

$$
\liminf _{n \rightarrow+\infty} \int_{S} f_{n}(s) \mu_{n}(d s)<+\infty, \quad-\infty<\int_{S} \liminf _{n \rightarrow+\infty, s^{\prime} \rightarrow s} f_{n}\left(s^{\prime}\right) \mu(d s)
$$

is violated, then (1.4) holds. So, we assume (4.10). The left inequality in (4.10) implies

$$
\liminf _{n \rightarrow+\infty} \int_{S} g_{n}(s) \mu_{n}(d s)<+\infty .
$$

Inequalities (4.9) and (4.11) imply that

$$
-\infty<\int_{S} \limsup _{n \rightarrow+\infty, s^{\prime} \rightarrow s} g_{n}\left(s^{\prime}\right) \mu(d s)<+\infty .
$$

In view of (4.12) and the right inequality in (4.10),

$$
\liminf _{n \rightarrow+\infty, s^{\prime} \rightarrow s} f_{n}\left(s^{\prime}\right)-\limsup _{n \rightarrow+\infty, s^{\prime} \rightarrow s} g_{n}\left(s^{\prime}\right) \leqslant \liminf _{n \rightarrow+\infty, s^{\prime} \rightarrow s}\left[f_{n}\left(s^{\prime}\right)-g_{n}\left(s^{\prime}\right)\right] \mu(d s) \text {-a.s. }
$$

and

$$
\begin{gathered}
\int_{S} \liminf _{n \rightarrow+\infty, s^{\prime} \rightarrow s} f_{n}\left(s^{\prime}\right) \mu(d s)-\int_{S} \limsup _{n \rightarrow+\infty, s^{\prime} \rightarrow s} g_{n}\left(s^{\prime}\right) \mu(d s) \\
\leqslant \int_{S} \liminf _{n \rightarrow+\infty, s^{\prime} \rightarrow s} h_{n}\left(s^{\prime}\right) \mu(d s),
\end{gathered}
$$

where $h_{n}(s)=f_{n}(s)-g_{n}(s), s \in S, n=1,2, \ldots$.

Let us apply Fatou's lemma for weak converging probabilities (see Theorem 1.1) to the sequence $\left\{h_{n}\right\}_{n \geqslant 1}$ of nonnegative $\overline{\mathbf{R}}$-valued measurable functions on $S$. Then

$$
\int_{S} \liminf _{n \rightarrow+\infty, s^{\prime} \rightarrow s} h_{n}\left(s^{\prime}\right) \mu(d s) \leqslant \liminf _{n \rightarrow+\infty} \int_{S} h_{n}(s) \mu_{n}(d s) .
$$

Since $-\infty<\liminf _{n \rightarrow+\infty} \int_{S} g_{n}(s) \mu_{n}(d s)<+\infty$ in view of (4.9), (4.11), and $g_{n} \leqslant f_{n}$, we have

$$
\begin{aligned}
\liminf _{n \rightarrow+\infty} \int_{S} h_{n}(s) \mu_{n}(d s) \leqslant & \liminf _{n \rightarrow+\infty} \int_{S} f_{n}(s) \mu_{n}(d s) \\
& -\liminf _{n \rightarrow+\infty} \int_{S} g_{n}(s) \mu_{n}(d s) .
\end{aligned}
$$

The following inequalities (4.14)-(4.16) and (4.12) imply (1.4) since

$$
\begin{aligned}
\int_{S} \liminf _{n \rightarrow+\infty, s^{\prime} \rightarrow s} f_{n}\left(s^{\prime}\right) \mu(d s)-\int_{S} \limsup _{n \rightarrow+\infty, s^{\prime} \rightarrow s} g_{n}\left(s^{\prime}\right) \mu(d s) \\
\quad \leqslant \liminf _{n \rightarrow+\infty} \int_{S} f_{n}(s) \mu_{n}(d s)-\liminf _{n \rightarrow+\infty} \int_{S} g_{n}(s) \mu_{n}(d s) \\
\quad \leqslant \liminf _{n \rightarrow+\infty} \int_{S} f_{n}(s) \mu_{n}(d s)-\int_{S} \limsup _{n \rightarrow+\infty, s^{\prime} \rightarrow s} g_{n}\left(s^{\prime}\right) \mu(d s),
\end{aligned}
$$

where the first inequality follows from (4.14) and (4.16), and the second inequality holds because of (4.9) and (4.12). Theorem 4.2 is proved.

$\mathrm{R}$ e $\mathrm{m}$ a $\mathrm{r} \mathrm{k} 4.2$. If $f_{n}(s) \geqslant K>-\infty$ for all $s \in S$ and $n=1,2, \ldots$, then assumptions (4.2) and (4.9) hold for $g_{n}(s)=K$ for all $s \in S, n=1,2, \ldots$. Thus Theorems 4.1 and 4.2 imply the validity of Fatou's lemma for setwise and weakly converging probability measures, if the functions $f_{n}$ are uniformly bounded from below. These facts also follow from (1.3) and Theorem 1.1, respectively.

$\mathrm{R}$ e $\mathrm{m}$ a $\mathrm{r} \mathrm{k}$ 4.3. Example 3.1 demonstrates that assumptions (4.2) and (4.9) are essential for Theorems 4.1 and 4.2 , respectively. 
$\mathrm{R}$ e $\mathrm{m}$ a r k 4.4. Theorem 1.1 yields that, for uniformly bounded above functions $\left\{g_{n}\right\}_{n \geqslant 1}$, assumption (4.9) in Theorem 4.1 is equivalent to

$$
\int_{S} \limsup _{n \rightarrow+\infty, s^{\prime} \rightarrow s} g_{n}\left(s^{\prime}\right) \mu(d s)=\lim _{n \rightarrow+\infty} \int_{S} g_{n}(s) \mu_{n}(d s)>-\infty .
$$

Indeed, applying Fatou's lemma for uniformly bounded from below functions $\left\{-g_{n}\right\}_{n \geqslant 1}$ (see Remark 4.2) we obtain the inequality

$$
\int_{S} \limsup _{n \rightarrow+\infty, s^{\prime} \rightarrow s} g_{n}\left(s^{\prime}\right) \mu(d s) \geqslant \limsup _{n \rightarrow+\infty} \int_{S} g_{n}(s) \mu_{n}(d s),
$$

that together with assumption (4.9) imply (4.17).

Corollary 4.1. Let $S$ be an arbitrary metric space, $\left\{\mu_{n}\right\}_{n \geqslant 1} \subset \mathbf{P}(S)$ converge weakly to $\mu \in \mathbf{P}(S)$, and $\left\{f_{n}\right\}_{n \geqslant 1}$ be a sequence of measurable $\overline{\mathbf{R}}$-valued functions on $S$. Then inequality (1.4) holds, if there exists a bounded above measurable $\mathbf{R}$-valued function $g$ on $S$ such that $f_{n}(s) \geqslant g(s)$ for all $n \geqslant 1$ and $s \in S$, and

$$
-\infty<\int_{S} \bar{g}(s) \mu(d s)=\lim _{n \rightarrow \infty} \int_{S} g(s) \mu_{n}(d s) .
$$

$\mathrm{R}$ e $\mathrm{m}$ a $\mathrm{r} \mathrm{k}$ 4.5. If function $g$ from Corollary 4.1 is upper semi-continuous (in particular, continuous), then assumption (4.18) has the following form:

$$
-\infty<\int_{S} g(s) \mu(d s)=\lim _{n \rightarrow \infty} \int_{S} g(s) \mu_{n}(d s) .
$$

In the following example functions $\left\{f_{n}\right\}_{n} \geqslant 1$ are unbounded from below and the assumptions of Theorem 4.1 are satisfied.

E x a m p l e 4.1. Let $S=\mathbf{Q}$ be the set of rational numbers with the metric $\rho\left(s_{1}, s_{2}\right)=\left|s_{1}-s_{2}\right|, s_{1}, s_{2} \in S$. We number the elements of $S=\left\{x_{i}\right\}_{i \geqslant 1}$ and the set $f_{n}=g_{n}=-n \mathbf{I}\left\{s \in D_{n}\right\}$, where $D_{n}=\left\{x_{1}, x_{2}, \ldots, x_{n}\right\}, n=1,2, \ldots$. Note that $\lim \sup _{n \rightarrow+\infty, s^{\prime} \rightarrow s} g_{n}\left(s^{\prime}\right)=0$ for any $s \in S$.

We consider an increasing sequence of natural numbers $\left\{k_{n}\right\}_{n \geqslant 1} \subset \mathbf{N}$ such that $k_{n} /\left(k_{n}+1\right) \notin D_{n}, n=1,2, \ldots$. Let us set

$$
\mu_{n}(B)=\mathbf{I}\left\{\frac{k_{n}}{k_{n}+1} \in B\right\}, \quad \mu(B)=\mathbf{I}\{1 \in B\}, \quad B \in \mathscr{B}(S), n=1,2, \ldots
$$

The sequence of probability measures $\left\{\mu_{n}\right\}_{n} \geqslant 1 \subset \mathbf{P}(S)$ converges weakly to $\mu \in \mathbf{P}(S)$. Moreover, assumption (4.9) holds. Therefore, Theorem 4.2 implies (1.4).

We remark that $g(s)=-\infty$ for all $s \in S$ for any function $g$ such that $g(s) \leqslant f_{n}(s)$ for all $n=1,2, \ldots$ and for all $s \in S$. Thus, $\bar{g}(s)=-\infty$ for all $s \in S$, assumption (4.18) does not hold, and Corollary 4.1 is not applicable to this example.

Acknowledgements. The authors thank Professor M.Z.Zgurovsky for initiating their research cooperation. The authors thank Dr. Huizhen Janey Yu for her useful remarks during the preparation of this work.

\section{REFERENCES}

1. Биллингсли П. Сходимость вероятностных мер. М.: Наука, 1977, 362 с.

2. Berberian S.K. Fundamentals of Real Analysis. New York: Springer-Verlag, 1999, $479 \mathrm{p}$.

3. Feinberg E. A., Kasyanov P. O., Zadoianchuk N. V. Average cost Markov decision processes with weakly continuous transition probabilities. - Math. Oper. Res., 2012, v. 37 , № 4 , p. 591-607.

4. Jaskiewicz A., Nowak A.S. Zero-sum ergodic stochastic games with Feller transition probabilities. - SIAM J. Control Optim., 2006, v. 45, № 3, p. 773-789. 
5. Royden H. L. Real Analysis. New York: Macmillan, 2nd ed., 1968.

6. Schäl M. 1993. Average optimality in dynamic programming with general state space. - Math. Oper. Res., 1993, v. 18, № 1, p. 163-172.

7. Serfozo $R$. Convergence of Lebesgue integrals with varying measures. - Sankhya: Ser. A, 1982, v. 44, №3, p. 380-402.

8. Ширяев А.Н. Вероятность. М.: МЦНМО, 2-е изд., 2012, 580 с.

Поступила в редакцию

17.V.2012

Исправленный вариант

15.V.2013

(c) 2013 г

MOHAMED N. M. A.*

\title{
MONTE CARLO SAMPLING OF MAXWELL AND GAUSSIAN DISTRIBUTIONS USING A SINGLE RANDOM NUMBER
}

\author{
Предложенная формула применяется к распределению Максвелла и \\ дает результаты с высокой точностью. \\ Ключевые слова и фразы: выборка Монте-Карло, распределение \\ Максвелла, гауссовское распределение.
}

1. Introduction. There are two basic methods of sampling in Monte Carlo method: the direct method and the rejection method [1], [2]. In direct method the random variable, $x$, is determined from the following equation:

$$
x=c^{-1}(r),
$$

where $c^{-1}(r)$ is the inverse function of the cumulative probability function $c(x)$ and $r$ is a random number uniformly distributed on $[0,1]$.

In order to apply the direct method, there are two conditions: 1) the probability density function (p.d.f.) can be integrated analytically and 2) the cumulative probability function must be invertible. If the p.d.f. does not satisfy these two conditions, the sampling usually is carried out using the rejection method. Some consider this method «crude» because random numbers are «wasted» unlike the direct method. It is particularly wasteful for «spiky» probability distribution functions [2]. Sampling from Gaussian distribution can be done by the Box-Muller-Wiener algorithm (BMW) [3]: if $r_{1}$ and $r_{2}$ are two random numbers uniformly distributed in the interval $[0,1]$, then the distribution can be sampled as

$$
x_{1}=\sqrt{2 \ln r_{1}} \sin \left(2 \pi r_{2}\right), \quad x_{2}=\sqrt{2 \ln r_{1}} \cos \left(2 \pi r_{2}\right),
$$

where $x_{1}$ and $x_{2}$ are random numbers distributed according to a Gaussian distribution of mean 0 and variance 1. Toral and Chakrabarti [4] showed that although the BMW algorithm is exact producing an unbiased Gaussian distribution and it is easily implementable on a vector computer, a serious disadvantage is that it is a rather slow algorithm. They reported that the rejection method is about 20 per cent faster than the pure BMW algorithm on scalar computers, but it has the disadvantage that it is not efficiently vectorizable. Other algorithms have been proposed [1] but they all share the unwanted feature of not being efficiently vectorizable. Toral and Chakrabarti mentioned an approximate method to generate Gaussian distributed random numbers as a more efficient method. More about nonuniform random variate generation is given in [5].

In general, Maxwell and Gaussian distributions with the p.d.f.'s

$$
\begin{aligned}
& p(x)=\frac{2 \sqrt{x}}{T^{3 / 2} \sqrt{\pi}} \exp \left(-\frac{x}{T}\right), \quad 0<x<\infty, \\
& p(x)=\frac{1}{\sigma \sqrt{2 \pi}} \exp \left(-\frac{x}{T}\right)\left[-\left(\frac{x-x_{1}}{\sqrt{2} \sigma}\right)^{2}\right], \quad-\infty<x<\infty,
\end{aligned}
$$

\footnotetext{
* Atomic Energy Authority, ETRR-2, Cairo, Egypt; e-mail: nmohamed@ictp.it
} 